\title{
High Precision Biaxial Solar Tracker
}

\author{
Z.Y. XIA, X.Y. CHEN, S.G. CHEN, L.H. HU, D.S. WU \& G.J. JIN \\ Mechanical and Electronical Engineering College, Fujian Agriculture and Forestry University, Fuzhou, \\ Fujian, China
}

\begin{abstract}
In this paper, a high precision biaxial solar tracker is designed, which is an application platform, can be used in solar photovoltaic projects and solar heat projects. Based on astronomy algorithm, we write a program in a Delta PLC to get the position of the sun by calculating its azimuth angle and altitude angle. Two DC motors is controlled by PLC to drive the solar tracker to rotate around both azimuth axis and altitude axis. Besides, three additional functions: hibernation, typhoon defender and snow cleaning, are designed to improve validity and reliability of the solar tracker. With Pro/E and ANSYS Workbench software, strength analysis in typhoon weather is made to assure the reliability of the solar tracker.
\end{abstract}

KEYWORD: high precision; solar tracker; astronomy algorithm; azimuth angle; altitude angle

\section{INTRODUCTIONS}

Solar energy technology has a generally problem of low conversion rate and low utilization rate. Photovoltaic panels and evacuated solar collector tubes are fixed installation in most cases, some solar devices can only rotate around one axis. Deviating from the sunlight direction in most time of daytime reduces the utilization rate of solar energy.[1-3] Photoelectric type solar tracking (passive tracking) device is easily affected by dust, floats and wet weather, then light sensors can't detect the sun position accurately,[4] which results in dysfunction of the solar tracker. Ordinary active solar tracking device is confined by the program, which always results in inefficient work.

In order to improve the utilization rate of solar energy, high precision biaxial solar tracker is designed. Based on astronomy algorithm and controlled by PLC, the tracker can make an all weather and real-time solar tracking motion. With protections of three additional functions, validity and reliability of the tracker is improved significantly.

\section{DESIGN PRINCIPAL OF THE SOLAR TRACKER}

\subsection{Description of the sun's position}

In astronomy, we often use celestial sphere model and horizontal coordinate system to describe the position of the sun.
As shown in Fig.1, the position of the sun is described by two angles - azimuth angle $(\mathrm{Az})$ and altitude angle (Alt). Az is the angle between projection line of sunlight on horizontal plane and south point. Alt is the angle between sunlight and horizontal plane.
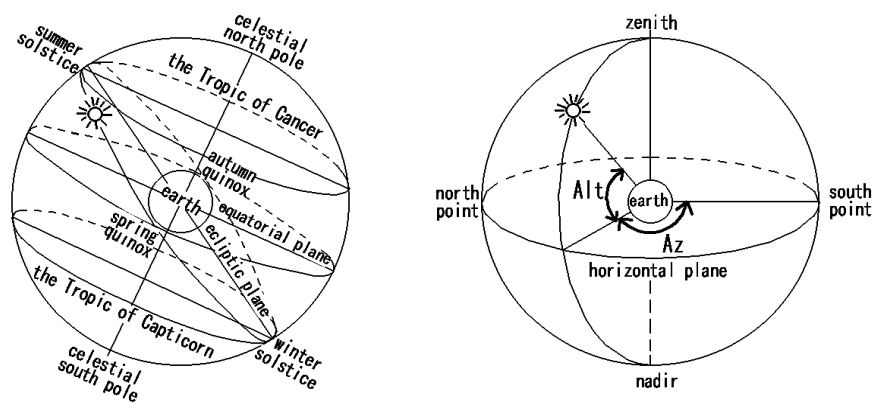

Fig.1 Celestial sphere mode and Horizontal coordinate system

\subsection{Calculation of the sun's position}

Azimuth angle and Altitude angle can be calculated by the following:[5]

$$
\begin{aligned}
& \sin (A 1 t)=\sin \Phi \sin \delta+\cos \Phi \cos \delta \cos \omega \\
& \sin (A z)=\frac{\cos \delta \sin \omega}{\cos (A 1 t)}
\end{aligned}
$$

where $\delta=$ declination of the sun; $\omega=$ hour angle; $\Phi=$ latitude .

Declination of the sun is calculated by the following:[6] 
$\delta=0.006918-0.399912 \cos \Gamma+0.070257 \Gamma-$

$0.006758 \cos (2 \Gamma)+0.000907 \sin (2 \Gamma)-$

$0.002697 \cos (3 \Gamma)+0.00148 \sin (3 \Gamma)$

where $\Gamma=[2 \pi(\mathrm{n}-1)] / 365 \mathrm{rad}$.

Hour angle equals the sum of local standard time and equation of time (EQT). Equation of time is essential to the calculation of accurate position of the sun. However, EQT values vary from day to day and year to year. EQT is calculated by the following: [7]

$$
\operatorname{EQT}(N)=\sum_{k=0}^{5}\left[A_{k} \cos \left(\frac{2 \pi k N}{365.25}\right)+B_{k} \sin \left(\frac{2 \pi k N}{365.25}\right)\right]
$$

where $\mathrm{N}=1 \sim 1461$. The value of $\mathrm{Ak}$ and $\mathrm{Bk}$ is referred to table 1:

Table 1. The value of $A_{k}$ and $B_{k}$.

\begin{tabular}{|c|c|c|}
\hline $\mathrm{k}$ & $\boldsymbol{A}_{\boldsymbol{k}} / \boldsymbol{h}$ & $\boldsymbol{B}_{\boldsymbol{k}} / \boldsymbol{h}$ \\
\hline 0 & $2.0870 \times 10^{-4}$ & 0 \\
1 & $9.2869 \times 10^{-3}$ & $-1.2229 \times 10^{-1}$ \\
2 & $-5.2258 \times 10^{-2}$ & $-1.5698 \times 10^{-1}$ \\
3 & $-1.3077 \times 10^{-3}$ & $-5.1602 \times 10^{-3}$ \\
4 & $-2.1867 \times 10^{-3}$ & $-2.9823 \times 10^{-3}$ \\
5 & $-1.5100 \times 10^{-4}$ & $-2.3463 \times 10^{-4}$ \\
\hline
\end{tabular}

\section{DESIGN OF THE SOLAR TRACKER}

\subsection{General design}

Hardware of the tracker mainly consists of control unit, executive body, mechanical structure and detection and protection, as shown in Fig.2:

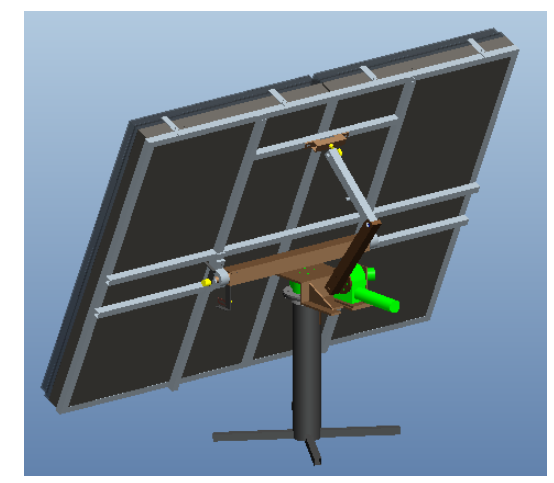

Fig.2 General structure of the solar tracker

Control unit is Delta DVP-40EH PLC. Cooperate with input ports X0 and X1, C251, C252, C253 and C254 can be used as high speed counter which its highest detected frequency is $4 \mathrm{KHz}$. With the high detected frequency, high-resolution encoder can be used to detect the angle's variation of azimuth actuator and altitude actuator. In this situation, high precision tracking and closed-loop control is easily accomplished.

Executive body consists of motivation and transmission. 24V DC motor and worm-gear reducer combine as one part to drive mechanical structures.
This integrating design could reduce the space and simplify the overall structure. Transmission parts are designed according to the principle of crank connecting rod mechanism. Crank drives connecting rod, connecting to the output of worm reducer. The end of the connecting rod hinges a panel-shaped steel structure, which is the solar application container $(4 \mathrm{~m} 2)$, built by angle irons and square steels. This makes it possible to change the altitude angle by pitching the container.

Detection and protection consist of photoelectric switch, incremental encoder $(3600 \mathrm{P} / \mathrm{R})$, wind speed sensor and proximity switch. Two encoders are separately installed on two rotary axes to detect the variation of azimuth angle and altitude angle. PLC real-time monitors the value of two encoders, and controls two DC motors to run once the current value of encoder differs from last value over 10 . At the position of $0^{\circ}$ azimuth angle and $0^{\circ}$ altitude angle, a proximity switch is installed to detect the origin. Limit switch also need to be installed on two axes to protect mechanical parts.

\subsection{Additional protections}

Hibernation is designed to determine whether to perform tracking with the use of photoelectric switch, which is used to test the brightness of environment. If the illumination intensity lowers to the threshold level (rainy day), PLC will receive this signal. Hibernation will be executed if this signal lasts for more than 10 minutes, which means tracking program will be terminated and tracker stays where it is. If the illumination intensity goes up to the threshold level, tracking program will be executed again. Hibernation reduces inefficient work time of the tracker, especially the long time wet weather in the south of China, and saves power.

Strong wind is the biggest threat to the tracker. With wind speed sensor, wind speed is easily read by PLC. When wind speed hits $17 \mathrm{~m} / \mathrm{s}$ (force 8 wind), altitude motor runs to where the container is in horizontal state $\left(90^{\circ}\right.$ altitude angle) and tracking program is terminated, which lowers the area of wind stress. When wind speed declines to $17 \mathrm{~m} / \mathrm{s}$ and this state lasts for more than 10 minutes, tracking program will be executed again. Typhoon defender could prevent strong wind, in case parts are damaged by strong wind, which improve the reliability and life time of the tracker.

Piezo-resistor is installed on the shady side of the container to detect the pressure of snow. According to literature, weight of $1 \mathrm{~cm}$ thick snow in $1 \mathrm{~m} 2$ is $1 \mathrm{~kg}$. The application area of the tracker is $4 \mathrm{~m} 2$, which means the thick of snow on the container increases $1 \mathrm{~cm}$, the pressure on it increases $4 \mathrm{~kg}$. If the pressure goes up to $4 \mathrm{~kg}$ and this signal can last for 1 minute at least. At the same time, wind speed is lower than $17 \mathrm{~m} / \mathrm{s}$ (in case strong wind leads to snow 
cleaning). The tracker rotates to $0^{\circ}$ altitude angle position to pour the snow. Snow cleaning could clear the snow in case of crush failure in wide-range and high intensity snow weather.

\subsection{Software design}

Software of the solar tracker is the program of astronomy algorithm. The program is divided into several subprograms: sun's position algorithm program, motor control program, monitoring program, alarm and protection program. The most important part is the sun's position algorithm program. According to equation (1), (2), (3), (4) and table 1, $\delta, \omega, \mathrm{Az}$ and Alt is calculated in every second by PLC, then the sun's position can be expressed by Az and Alt. Compare to the last time value of $\mathrm{Az}$ and Alt respectively, motor control subprogram is called once difference value rise to 10 ( $1^{\circ}$ deviation). Monitoring program is separated into 3 parts: illumination intensity data acquisition, wind speed value processing and snow pressure data processing.

\section{WORKING PRINCIPLE OF THE SOLAR TRACKER}

Once it operates, photoelectric switch detects whether the illumination intensity is suitable to start tracking. If the intensity is enough, tracking program will be executed. In one word, tracking is changing the 2 angles: Az and Alt. The executive body runs when the deviation value of current time and last time of these two angles reaches to 10 respectively. Motors don't stop until the deviation value equals 0 , where the container faces to the sun. The figure of working principle is shown:

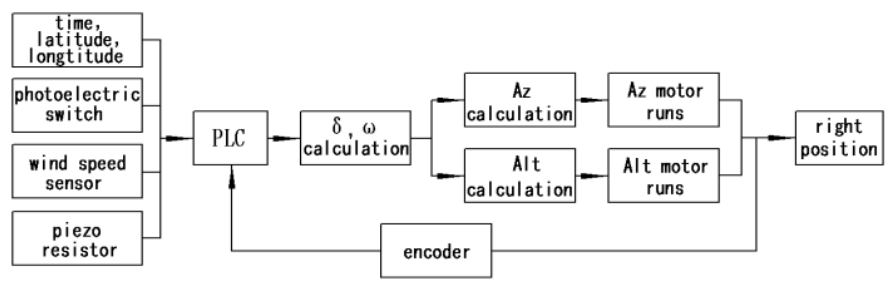

Fig.3 working principle of the solar tracker

Control system consists of Delta PLC, Delta human-machine interface, encoder, photoelectric switch, piezo-resistor, wind speed sensor. Humanmachine interface is used to set original time, geography information and display the running state of the tracker, such as: when tracking starts and stops, when typhoon defender and snow cleaning executes, and alarm information. The work time of the tracker is from sunrise to sunset and the working process of it is shown as following:

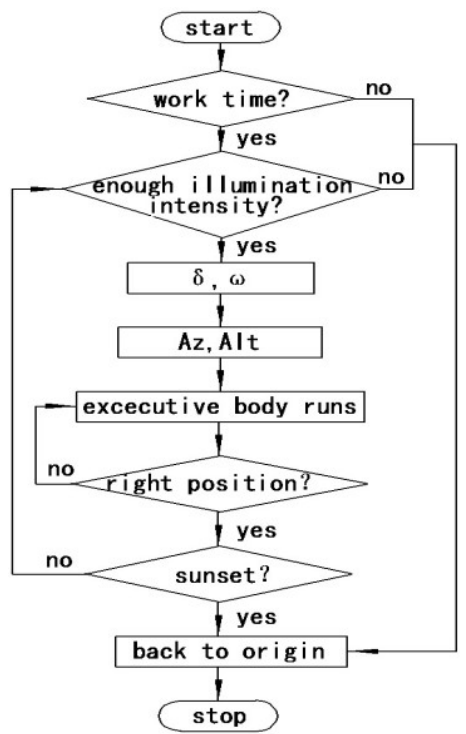

Figure.4 Work flow chart of the solar tracker

\section{STRENGTH ANALYSIS}

Strong wind weather is the biggest enemy to the solar tracker. With the help of finite elements analysis software ANSYS Workbench, the strength analysis is accomplished. Dynamic wind pressure is calculated by:

$p=0.5 \cdot r \cdot v^{2} / g$

where $p\left[\mathrm{kN} / \mathrm{m}^{2}\right]=$ wind pressure; $v[\mathrm{~m} / \mathrm{s}]=$ wind speed; $r\left[\mathrm{kN} / \mathrm{m}^{3}\right]=$ wind unit weight, $\mathrm{r}=0.01225 \mathrm{kN} / \mathrm{m}^{3}$.

An estimating formula about wind pressure is:

$p=v^{2} / 1600$

When Alt equals $0^{\circ}$, the tracker bears the largest pressure, and area where mainly endure pressure locates main support. The speed of force 12 wind is $32.7 \mathrm{~m} / \mathrm{s}-36.9 \mathrm{~m} / \mathrm{s}, \quad p$ roughly equals $0.85 \mathrm{kN} / \mathrm{m}^{2}$ according to equation (6). Fix the 4 bottom supporting rods in Workbench, and apply $1.7 \times 10-3$ MPa pressure on each piece of the container (2 pieces, each piece is $2 \mathrm{~m}^{2}$ ). The total deformation chart and von-Misses stress chart are shown in Fig.5 (application units has been hidden).
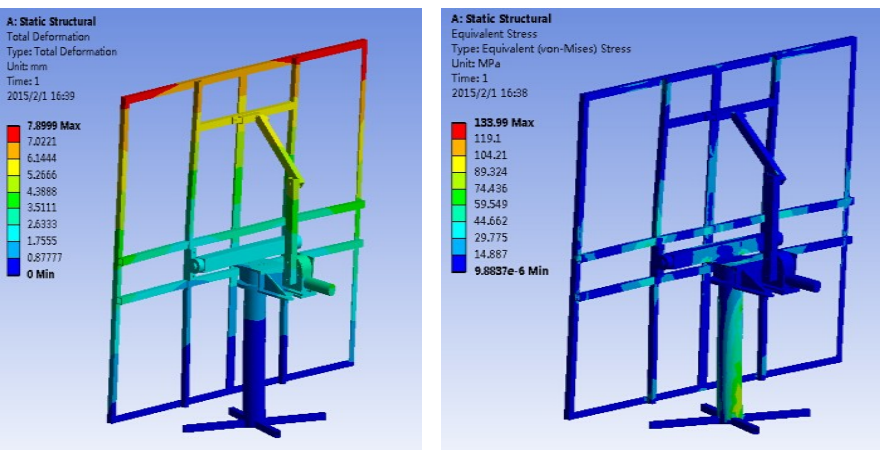

Fig. 5 Deformation and von-Mises stress chart $\left(\mathrm{Alt}=0^{\circ}\right)$

Under the influence of strong wind, deformation maximum, $7.9 \mathrm{~mm}$, locates the edge of the container. 
Stress maximum, 134MPa, locates several parts of the tracker: beam, framework of the container, connection of reducer and beam, connection of main support and bottom supporting rods and each hinge joint.

Once strong wind comes when it is tracking, Alt motor runs to where Alt equals $90^{\circ}$. A force 12 wind analysis is done in this condition, which stress area has changed to the whole tracker. As Fig.6 shows, deformation maximum, $2.9 \mathrm{~mm}$, locates the edge of the container, connection of beam and reducer. Stress maximum, $51.5 \mathrm{MPa}$, locates the transition of reducer and beam. However, because of reducing of stress area, deformation maximum and stress maximum decrease significantly. This could verify the importance of the typhoon defender and also validate the reliability of the tracker in strong wind weather.
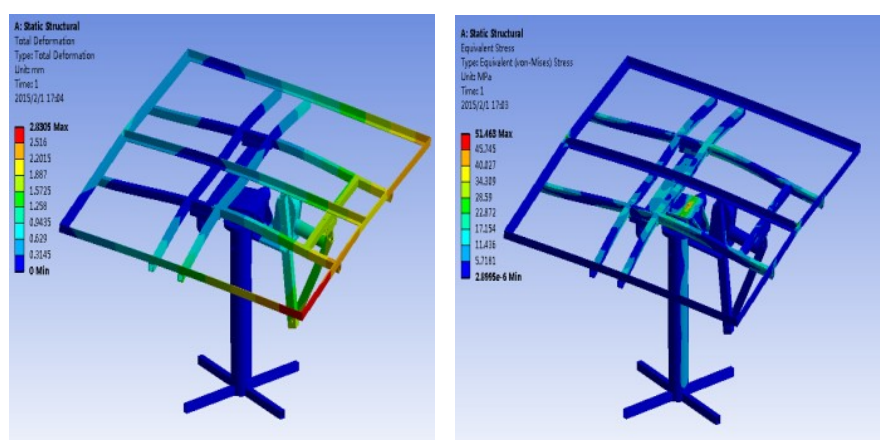

Fig.6 Deformation and von-Mises stress chart $\left(\mathrm{Alt}=90^{\circ}\right)$

\section{CONCLUSION}

The solar tracker makes it possible for high precision biaxial tracking and the problem of photoelectrical tracking failure in rainy weather and harsh environment is solved by means of astronomy algorithm. With high accuracy encoder, closed-loop control is accomplished to feed back the real-time value of azimuth angle and altitude angle, which also improves the precision of tracking and solar utilization. Comprehensive protective functions are designed additionally on the basis of ordinary solar trackers to improve the validity and reliability of the solar tracker. The so-called solar tracker in this paper is a platform for solar photovoltaic projects and solar heat projects. High precision biaxial solar tracker expands the application scope of solar energy and promotes the solar energy industry.

\section{REFERENCES}

[1] Gan Yi, Wang Zijian, Zeng Lecai, et al. 2013. Design of a solar tracking controller based on the 89C51 microcontroller. Energy Research and Information, 29(3): 173-178.

[2] Ma jian, Xiang ping. 2008. High Precision Computation for Sun Position and Its Application in Solar Power Generation. Water Resources and Power, 26(2): 201-204.

[3] Ding Tingting, Zhu Xuemei. 2012. Research on Suntracking Control System in Solar Photovoltaic Power and Its Design. Jiangsu Machine Building and Automation, 41(6) :200-205.

[4] Bian Wanrong, Zhao Hui, Yang Yun, et al. 2013. Active Solar Tracking System Design. Microcontrollers and Embedded Systems, 13(2): 55-58.

[5] Chen Shiguo, Chen Xueyong, Lin Fucai, et al. 2014. Design of solar tracking control system. Journal of Fujian Agriculture and Forestry University (Natural Science Edition), 43(2):215-218.

[6] Spencer J.W. 1971. Fourier series representation of the position of the sun. Search, 2(5):172.

[7] Lamm L.O. 1981. A new analytic expression for the equation of time. Solar Energy, 26(5):465. 